\author{
Tihomir Mihalić ${ }^{1}$ \\ e-mail: tihomir.mihalic@fsb.hr \\ Zvonimir Guzović \\ e-mail: zvonimir.guzovic@fsb.hr \\ Faculty of Mechanical Engineering \\ and Naval Architecture, \\ University of Zagreb, \\ I.Lučića 5 , \\ 10000 Zagreb, Croatia \\ Andrej Predin \\ Faculty of Energy Technology, \\ University of Maribor, \\ Hočevarjev $\operatorname{trg} 1$, \\ 8270 Krško, Slovenia \\ e-mail: andrej.predin@uni-mb.si
}

\section{Performances and Flow Analysis in the Centrifugal Vortex Pump}

Improvements to the characteristics of a centrifugal pump through the addition of a vortex rotor were investigated both experimentally and with computational fluid dynamic (CFD) analysis. The idea behind that improvement is in creating so-called coherent structures of eddies and turbulence in the peripheral area of the vortex rotor mounted at the back side of centrifugal rotor. Research on the energy transformations in the centrifugal vortex pump in this work was carried out using numerical simulations of the flow in the centrifugal and the centrifugal vortex pump. Measurements of relevant parameters that describe the performance of pumps, at their physical models, were gained from experiments. The measurement results were used as experimental validation of numerical simulations. In contrast, flow visualization derived from the numerical simulation was used to interpret measurements. In deriving the experimental procedure, special care was taken with the flow measurements. The reason for this is in the fact that the flow measurements had the biggest influence on the overall measurement uncertainty. However, flow measurements were the most demanding with regards to the experiment design and in taking the measurement readings. This experimental-CFD research made it possible to undertake an assessment of vortex rotor contribution on the head of the centrifugal vortex pump. The influence of the vortex rotor on the efficiency of the centrifugal vortex pump was investigated by comparing it with the efficiency of the centrifugal pump with the same geometry. An analysis of the flow structure was conducted in order to better understand the energy transformations that are the result of the interaction between the flow from the channels of the centrifugal part of the centrifugal vortex rotor and vortices formed at the vortex part of the centrifugal vortex rotor as well as their interactions with the stator. It was shown that this additional energy significantly increases pump head while increasing pump stability. This synergetic work has demonstrated that while vortex rotor gives additional energy to the fluid particles, that did not enter stator due to the energy lack by changing their momentum; at the same time, some of the kinetic energy contained in the vortex rotor induced vortices is also added to those fluid particles. [DOI: 10.1115/1.4023198]

Keywords: centrifugal vortex pump, coherent structures, rotor fluid interaction, unsteady flow, $C F D$

\section{Introduction}

One way of improving the characteristics of centrifugal pumps is by adding a vortex rotor to the centrifugal rotor by which energy from induced vortices at the vortex rim is added to the fluid energy gained in the centrifugal rotor, as demonstrated by Refs. [1-4], Fig. 1.

Figure 2 shows a cross section of the centrifugal vortex pump stage with annotated major components. On the periphery of the disc (1) of the centrifugal rotor (2) on the stator side (4), the vortex rim (rotor) is installed (3). There is a gap between the stator cover and vortex radial vanes that, together with the gap between centrifugal vanes and the stator entrance, forms a peripherallateral annular vortex chamber.

The resulting additional kinetic energy induced at the vortex rim transforms to the head $H_{4}$, which is added to the bulk head (pressure) obtained in the centrifugal rotor of the centrifugal vortex pump $H_{\text {cen }}$ as shown in Eq. (1).

$$
H=H_{\text {cen }}+H_{4}
$$

Experiments alone do not provide all the necessary information about the structure of the energy conversions. However, unsteady phenomena in the form of interactions of rotor and stator, and other flow instabilities continue to make accurate prediction of the

\footnotetext{
${ }^{1}$ Corresponding author

Manuscript received August 5, 2012; final manuscript received November 28 , 2012; published online January 18, 2013. Assoc. Editor: Chunill Hah.
}

pump characteristics a difficult and time-consuming task, which still requires validation and verification of the numerically obtained results. Therefore, the use of both methods, computer simulations (CFD) together with experiments, can provide insight

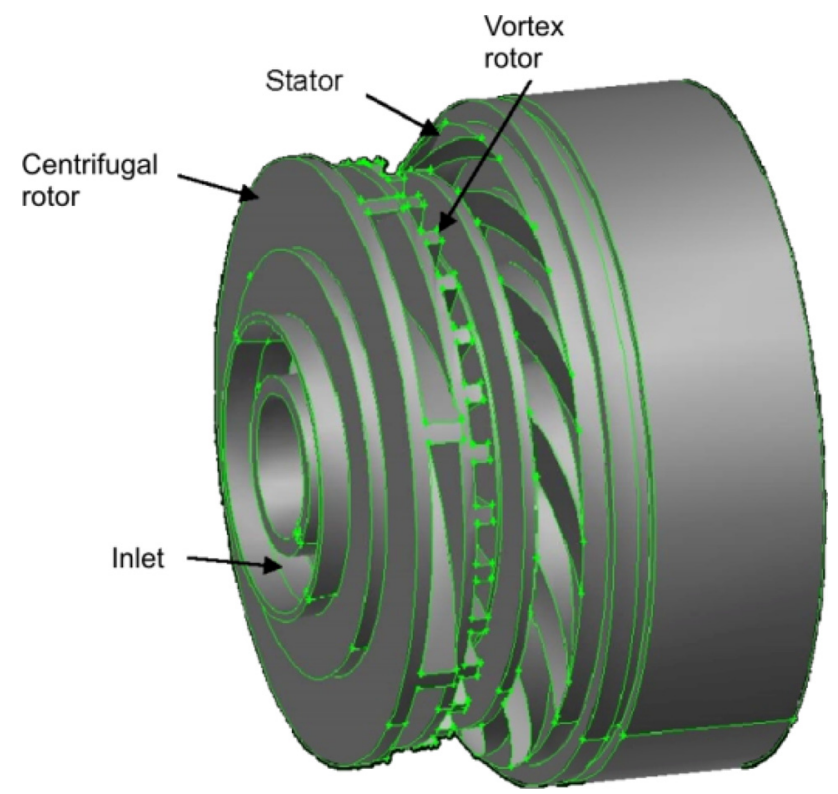

Fig. 1 Centrifugal vortex pump stage 


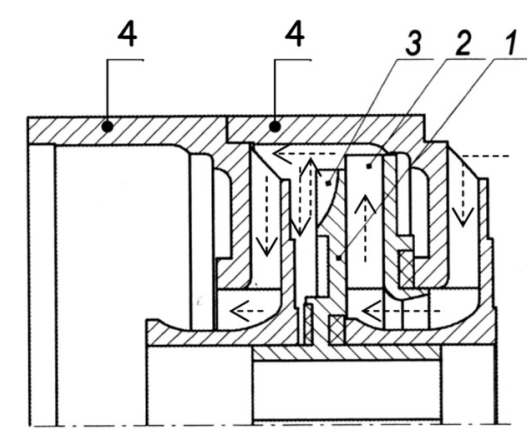

Fig. 2 Cross section of the centrifugal vortex pump stage (dashed arrow lines shows fluid flow)

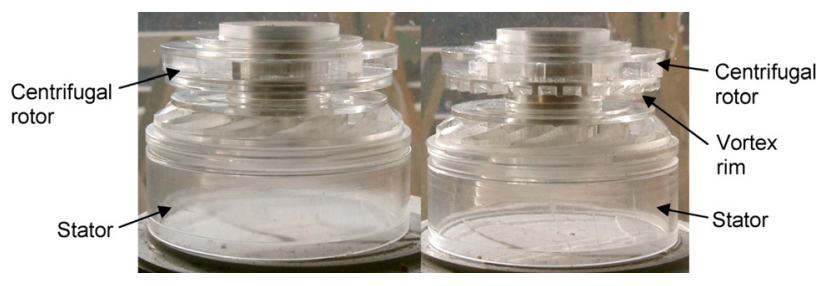

Fig. 3 Centrifugal and centrifugal vortex pump stage made of Plexiglas for experimental research

into the structure of flows and the quantification of parameters, which helps in understanding the processes of energy conversions within this type of machine.

During this research, physical models of the centrifugal and centrifugal vortex pump were made, which correspond to numerical models and the experimental line with the respective equipment that allowed the change of angular velocity, the setting of the working point and the measuring of the current flow, increment of pressure and propulsion power. The numerical simulations of unsteady flow in centrifugal pumps and centrifugal vortex pumps were conducted using a commercial software package which is based on the control volume method.

The objectives of this research were to clarify the mechanisms of energy conversion in a centrifugal vortex pump and to confirm the positive impact of a vortex rim. To do that, it was necessary to research the flow structures occurring in the peripheral-lateral annular vortex chamber and the interaction of this flow with vortex rotor and with the stator.

\section{Experimental Model of Centrifugal Vortex Pump}

The rotor and stator, machined from transparent plexiglass, were also embedded in a transparent casing of plexiglass to enable flow visualization, both in the passages of the rotor and the stator and in the zone of special interest for research, i.e., the vortex chamber, where the vortex rim is a located (Fig. 3).

The experimental assembly, Fig. 4, had valves used for regulating measurements and pressure transducers connected to a signal acquisition module. The experimental assembly is made in ac-

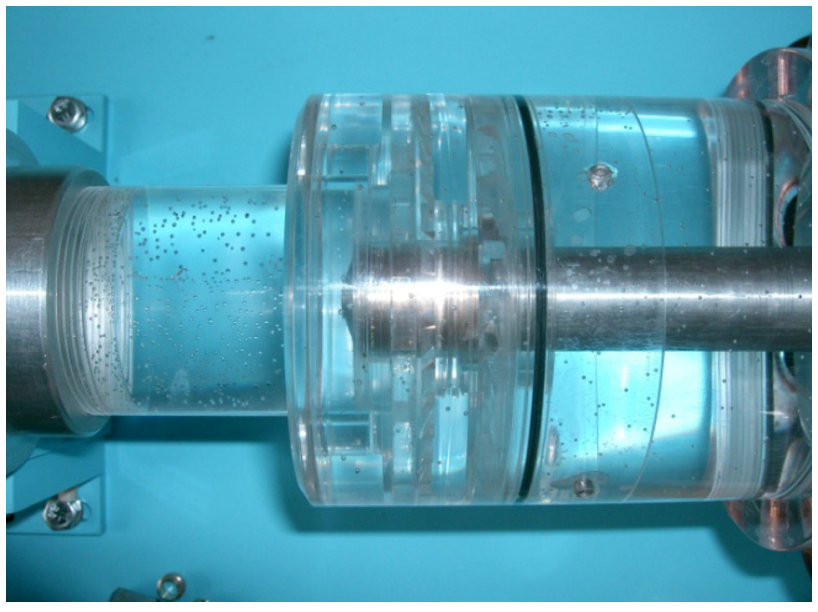

Fig. 5 Pump stage housing

cordance with the following standards: ISO 5167-1, 4:2003 and ISO / TR 15377:2007.

The housing of the experimental pump stages was designed to allow rapid exchanging of different pump rotors (Fig. 5). Furthermore, the housing provides all the necessary seals between the rotor and the suction zone, the front plate and housing and the stator and housing.

\section{Flow Measurement}

Venturi flow meters achieves a very high accuracy of $< \pm 0.5 \%$, as demonstrated by Franjić [5] and Goldstein [6]. Because of that, but primarily due to the fact that its measurement uncertainty depends on the measurement uncertainty for linear measurements of its dimensions (diameter of the sections at the pressure measurement stations) and pressure measurement uncertainty, a venturi flow meter has been designed and produced for the purpose of this research (Fig. 6)[7].

When designing venturi flow meter, special attention was paid to the collectors for measuring pressure. To obtain a more accurate pressure that exists in the measuring section (nullifying the impact of separation, turbulence, and other instabilities), the pressure is taken at four points along the rim section, and it is integrated with the collector and measured at one point, as shown by Refs. [8-10].

\section{Numerical Model of Centrifugal Vortex Pump}

Numerical simulations of the unsteady flow in centrifugal, centrifugal vortex pumps and the vortex part of centrifugal vortex pumps were carried out using Ansys FLUENT 14.0. The simulations include a whole centrifugal and centrifugal vortex pump stage including the suction and discharge pipes (Fig. 7). The CFD model neglects the gap between the rotor and housing at the inlet side and also presumes that the sealing between the rotor and stator, the rotor and the suction zone is perfect. Furthermore, the CFD model neglects friction losses due to wall roughness.

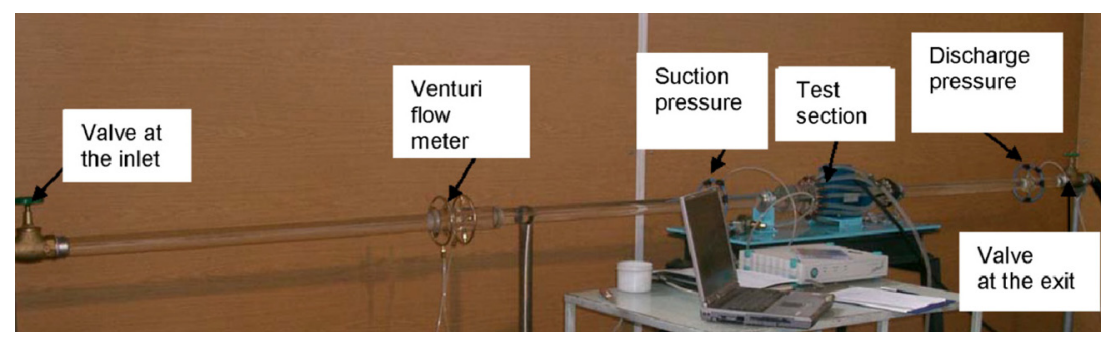

Fig. 4 Experimental assembly 


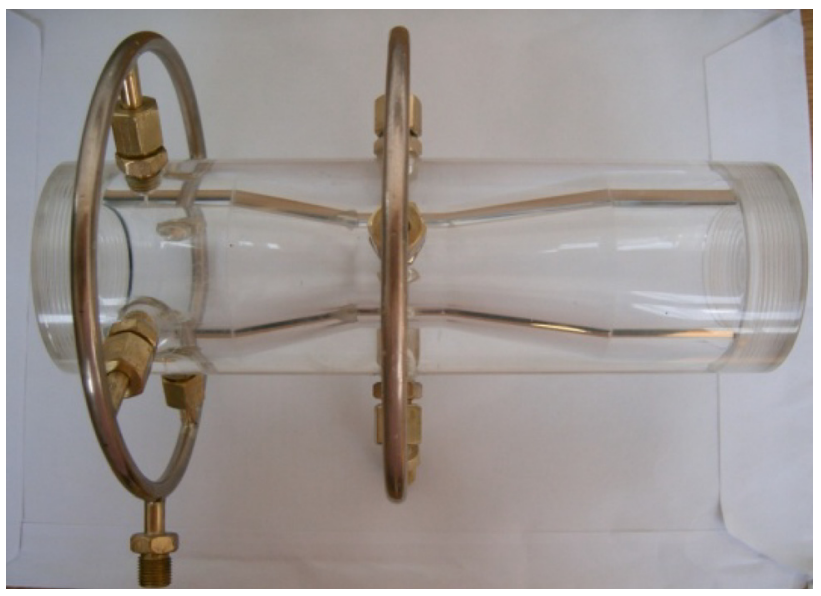

Fig. 6 Designed venturi flow meter

On the left side of Fig. 7, the meshed stator with the outlet is shown, while the meshed rotor with the inlet pipe is shown on the right side.

In the CFD model, the rotor rotates unrestrained, and is connected to the rest of the domain with a sliding mesh boundary condition. Calculation of the rotor (Fig. 8) takes place in a noninertial, rotating coordinate system. The output tube is connected with the output from the stator with the interface boundary condition. This allows the output tube to have coarser mesh, thus saving computational time (Fig. 9).

The entire continuum was meshed with three different meshes of control volumes (CV) as shown in Table 1 .

Given that, the research was conducted at the rotor angular speed $n=2910 \mathrm{~min}^{-1}$, simulations were performed with a time step of $8 \times 10^{-5} \mathrm{~s}$. Within the single time step, the rotor is turned by $1.5 \mathrm{deg}$. The time step was chosen after research for a suitable time step with respect to convergence. Selected time step of $8 \times 10^{-5}$ for these simulations ensured that the mean Courant number (CFL) was 0.5179 . For the working fluid, water of standard properties at $25^{\circ} \mathrm{C}$ was used.

Turbulence was modeled using a hybrid DES SST model (detached eddy simulations), as demonstrated by Spalart [11] and Travin et al. [12].

Boundary conditions were set far enough from the pump stage so that their impact on the flow could be neglected. At the entrance to the domain, a pressure-inlet boundary condition was used, while at the exit from the domain, an outlet-vent boundary condition was used because it allows adjustment of the loss coefficient at the exit.

The boundary condition outlet vent is defined by pressure loss (attenuation) that is proportional to dynamic pressure, Eq. (2),

$$
\Delta p=k_{L} \frac{1}{2} \rho v^{2}
$$

The outlet vent was also chosen because it best describes the physics of this research, in which the flow is regulated by the valve at the exit, from fully open to fully closed. The loss coefficient $k_{L}$ in the boundary condition outlet vent (Eq. (2)) is a number, and the whole simulation was conducted with $k_{L}=0$ (fully open valve), 2, 5, 10, 20, 60, and 300 (fully closed valve), as shown in the FLUENT manual [13].

\section{Results of the Numerical and Experimental Research of Centrifugal Vortex Pump}

5.1 Comparison of $Q-H$ Characteristic of Centrifugal and Centrifugal Vortex Pump. Figure 10 shows the $Q-H$ characteristics of a centrifugal pump stage and the $Q-H$ characteristics of a hybrid centrifugal vortex pump stage. It is evident that the characteristics of the centrifugal vortex pump smoothly follow the
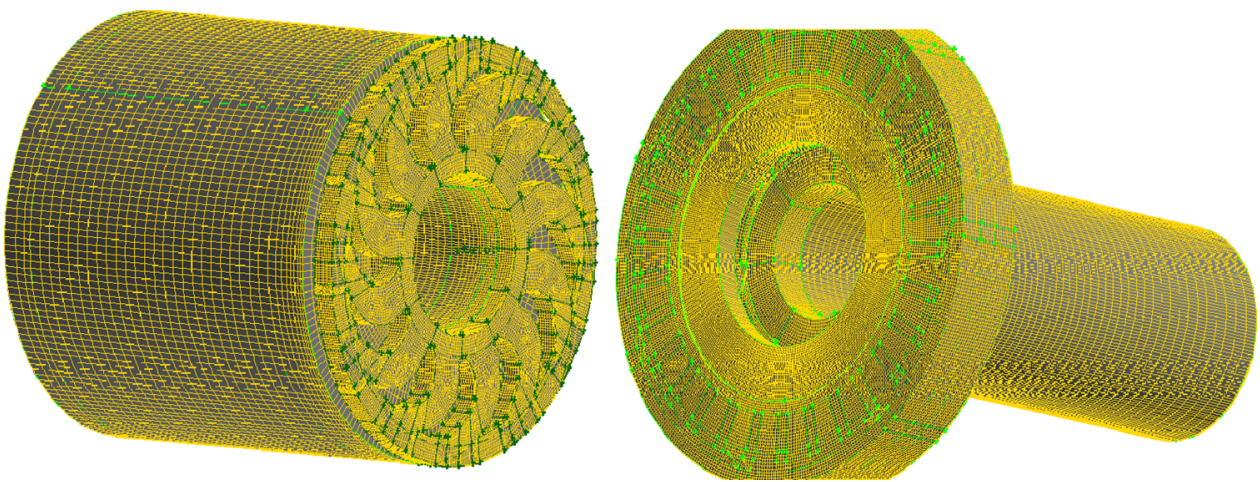

Fig. 7 Control volume mesh of centrifugal vortex pump stage
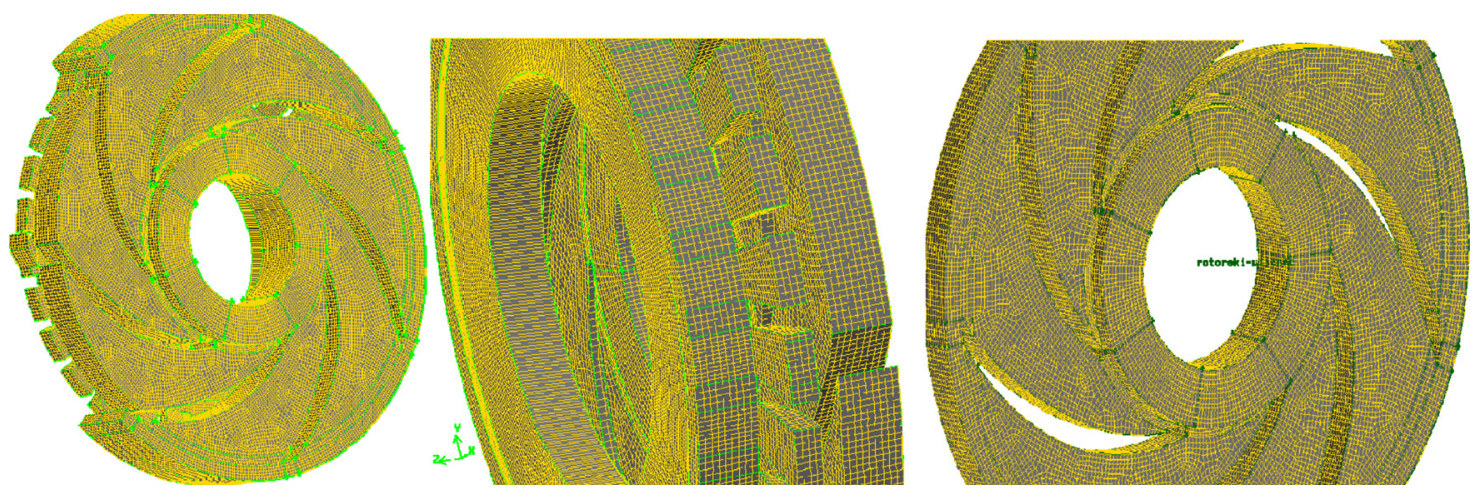

Fig. 8 Structured mesh of rotor 


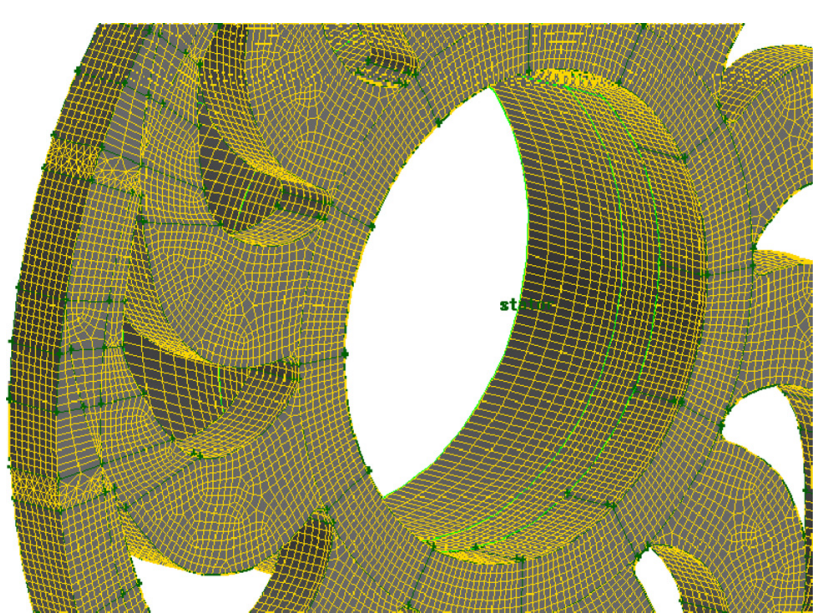

Fig. 9 Structured mesh of stator

Table 1 Comparison of used meshes

\begin{tabular}{lrrcc}
\hline \hline & \multicolumn{1}{c}{$\begin{array}{c}\text { No. } \\
\text { of CV }\end{array}$} & \multicolumn{1}{c}{$\begin{array}{c}\text { No. } \\
\text { of nodes }\end{array}$} & $\begin{array}{c}\text { Largest CV } \\
\text { edge }(\mathrm{mm})\end{array}$ & $\begin{array}{c}\text { \% of } \\
\text { hexahedral CV }\end{array}$ \\
\hline First mesh & 962,159 & 108,640 & 0.5 & 85 \\
Second mesh & $1,864,399$ & $2,007,183$ & 0.4 & 85 \\
Third mesh & $3,340,658$ & $3,573,140$ & 0.3 & 85 \\
\hline \hline
\end{tabular}

characteristics of centrifugal pump from the maximum flow to the flow of $1.22 \mathrm{l} / \mathrm{s}\left(105 \mathrm{~m}^{3} /\right.$ day $)$. After the flow of $1.22 \mathrm{l} / \mathrm{s}$ (105 $\mathrm{m}^{3} /$ day), the characteristics of the centrifugal vortex pump has a positive slope as a characteristic of vortex pumps (Dochterman [14]), in contrast to the characteristics of centrifugal pump, which grows slower than the characteristics of the centrifugal vortex pump to its maximum at flow rate of $0.751 / \mathrm{s}\left(65 \mathrm{~m}^{3} /\right.$ day $)$, and then gains a negative slope. This form of performance curve (falling steeply after its maximum) represents the instability of the pump, because the pump can generate two different flow rates at a given head, left and right from the maximum $Q-H$ characteristic curve, as pointed out by Lobanoff and Ross [15].

Adding a vortex rotor to the centrifugal pump stage provided a steeply sloping $Q-H$ characteristic pump, which ensures the stability of its operation. This confirmed one of the positive roles of the vortex rim in the centrifugal vortex pump stage.

5.2 Quantification of the Portion of Head Generated by Vortex Rotor in the Head of a Centrifugal Vortex Pump. The contribution of eddy processes on the amount of the head and the

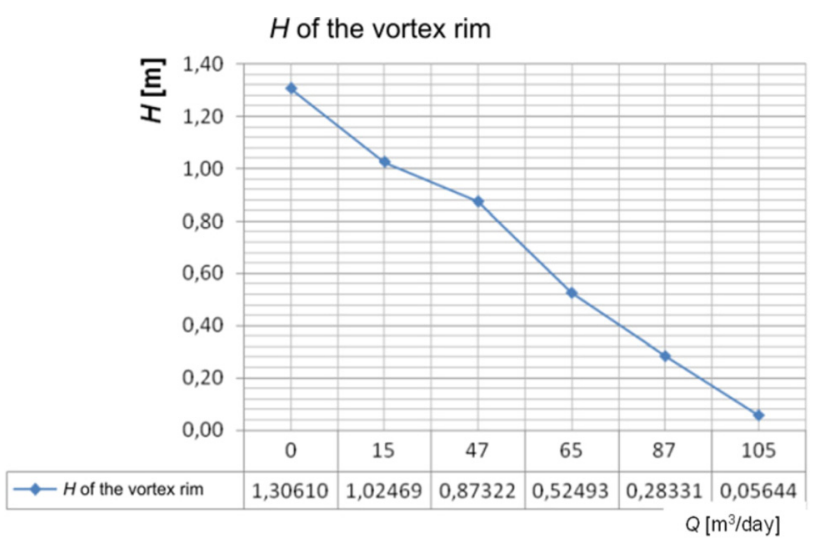

Fig. 11 Contribution of the energy fluxes from the vortex rim to the increase of head $\mathrm{H}, n=2910 \mathrm{~min}^{-1}$

corresponding increase in pressure depends on the flow rate, and the given flow rate depends on the magnitude of the axial component of absolute velocity of the fluid from the discharge of the centrifugal rotor to the entrance in the diffuser channels. The lower the flow rate, and the smaller the axial velocity component are, the greater the number of the vortex rim passages participating in the change of momentum between the fluid contained in these passages at that instant of time and the main stream of the fluid from the centrifugal rotor to the diffuser, as demonstrated by Mihalić et al. [16].

Figure 11 clearly shows that the vortex rim in the centrifugal vortex pump stage begins to increase the amount of the head from the flow rate of $1.22 \mathrm{l} / \mathrm{s}\left(105 \mathrm{~m}^{3} /\right.$ day $)$ to the zero flow rate, which is very convenient, because if there is a higher demand on the pump, so as to overcome the greater resistance, the vortex rim is increasingly helping the pump. These are experimental results.

The swirl effect achieved by the vortex rim increased the magnitude of the head of the plain centrifugal pump stage for a maximum of $23.13 \%$ (average increase of $11.64 \%$ ).

5.3 Characteristics of the Vortex Part of a Centrifugal Vortex Pump. Figure 12 shows the head of a vortex part of a centrifugal vortex pump derived with a CFD simulation of the researched centrifugal vortex pump by taking out the centrifugal rotor. As evident from the figure, the vortex rotor produced a maximum of $0.63 \mathrm{~m}$ of head, while operating in synergy with the centrifugal rotor; it generated a maximum of $1.306 \mathrm{~m}$ of head as can be seen from Fig. 11. Therefore, the vortex rotor, in synergy with the centrifugal rotor, generates a double head, as opposed to acting alone. The reason for this is in fact that when it works in synergy with the centrifugal rotor, it affects the secondary stream

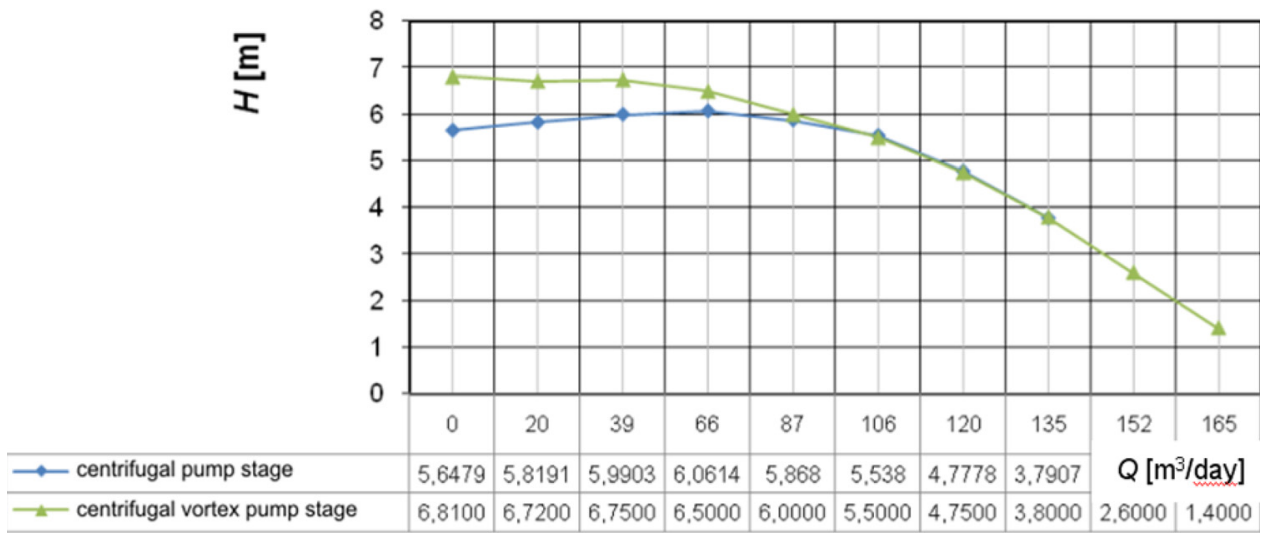

Fig. 10 Comparison of Q-H characteristics of centrifugal vortex and centrifugal pump stage, $n=2910 \mathrm{~min}^{-1}$ 
Q-H of the vortex part of the centrifugal vortex pump stage

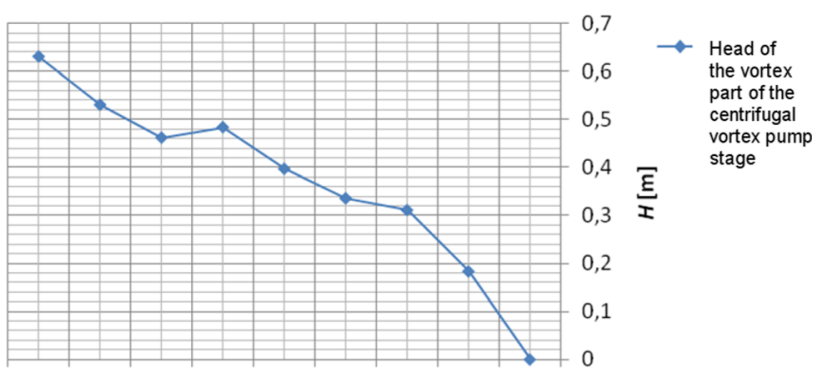

$0,00 \quad 10,2711,9421,5323,9927,5030,7838,0543,45 \quad Q\left[m^{3} /\right.$ day $]$

Fig. 12 Head of vortex part of centrifugal vortex pump, $n=2910 \mathrm{~min}^{-1}$

which already gained a certain amount of energy in the centrifugal rotor (see Fig. 15 and Fig. 16).

From Fig. 12, it can be seen that when a vortex rotor is acting alone, it create a steep drop-down characteristic and a low fluid flow rate. The maximum consumption of power when the vortex rotor works alone is $33.66 \mathrm{~W}$, while the maximum power brought to centrifugal vortex pump stage is $108.25 \mathrm{~W}$. Therefore, the vortex rotor working alone, in this configuration, has a very low efficiency.

5.4 Comparison of the Efficiency of Centrifugal Vortex and Centrifugal Pump Stage. Pressure transducers for measuring pressure difference from the pump intake and outlet, together with installed torque meter between the pump stage and operating motor, make it possible to gain pump efficiencies at different flow rates.

Figure 13 shows a comparison of efficiency curves of the centrifugal vortex and centrifugal pump stage researched in this work. It can be assumed that both curves show a greater efficiency than would be present with the utilization of actual pumps. The reason for this is that the numerical models do not take into account friction losses due to wall roughness, and losses due to fluid flow through clearances as shown by Gülich [17]. However, we assumed that the numerical models of real pump geometry will give efficiency curves with the realistic trends and mutual correlations.

A comparison of the efficiency curves (Fig. 13) shows that at high flow rates (low loss coefficient $k_{L}=0$ to 10), the centrifugal pump demonstrated higher efficiency than the centrifugal vortex pump. The vortex rotor at high flow rates does not get enough fluid from the main flow (coming from the centrifugal rotor, Fig. 15) so it does not increase the head (Fig. 10), while at the same time it contributes significantly to the increase of entropy. In that working range, the vortex rotor only creates turbulence, and its kinetic energy is converted into a losses. Thus, in this working range, the vortex rotor is a "parasitic part" in the centrifugal vortex pump.

\section{Efficiency of the centrifugal vortex and centrifugal pump stage}

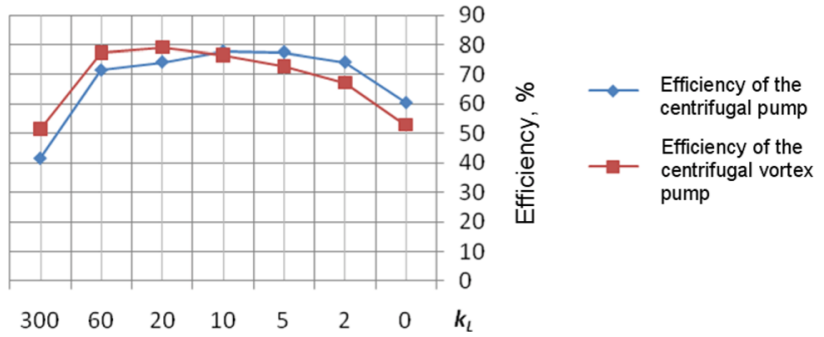

Fig. 13 Comparison of the efficiency curve of centrifugal vortex and centrifugal pump

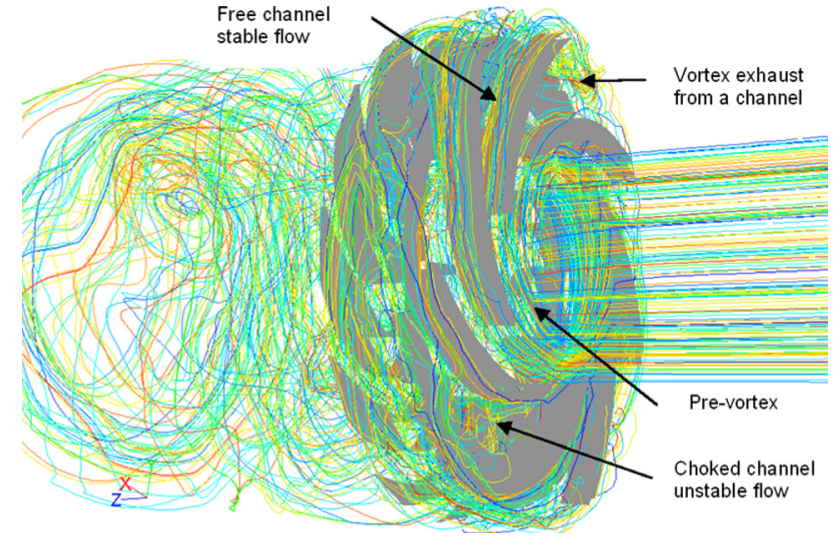

Fig. 14 Pathlines in the centrifugal vortex pump $k_{L}=60$

In contrast, at low flow rates (high loss coefficient $k_{L}=10$ to 300 ) the efficiency of the centrifugal vortex pump is greater than that of the centrifugal pump. Operating at this working regime of the low flow rate, the vortex rotor grips a sufficient amount of fluid from the main stream and contributes to increasing the level of head (Fig. 10). Its kinetic energy is not transferred entirely to the creation of fluid turbulence, but a part of its kinetic energy is used to create a coherent flow structures and to accelerate the flow of secondary stream (Sec. 5.6).

It is also evident from the Fig. 13 that the best efficiency point of the centrifugal vortex pump is shifted to the left (to the smaller flow rates, and higher head) in comparison to the centrifugal pump.

5.5 Analysis of the Pathlines in the Centrifugal Vortex Pump. Figure 14 shows the flow through a centrifugal vortex pump; here, the flow structures can be observed. It can be seen that the flow through inlet pipe is even, steady, and nearly laminar with pre vortex at the entrance to the rotor. It is evident that after the vortex rotor, flow has substantially more coherent structures (Fig. 18). In the stator, flow becomes less chaotic, but still there are vortices which are now fewer and with larger diameters. From the figure, it can also be observed that some centrifugal rotor channels are choked with vortex while in others there are no vortices whatsoever. Furthermore, in other channels, vortex is moving out from them. This phenomenon of the vortex appearing to be one and the same vortex traveling centrifugally from one rotor channel to another represents a traveling vortex instability and loss of energy, and is called "flow cutoff" or "rotating stall," as demonstrated by Matijašević et al. [18].

5.6 Analysis of the Secondary Fluid Flow. In Fig. 15, the velocity vectors in the axial plane of the researched centrifugal vortex pump are shown. The formation of the main fluid flow coming from the centrifugal rotor and a secondary flow that

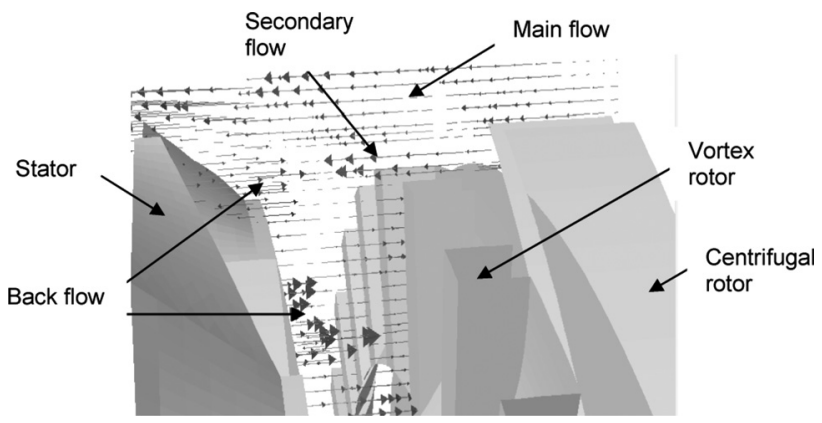

Fig. 15 Main and secondary flow of fluid, $k_{L}=60$ 


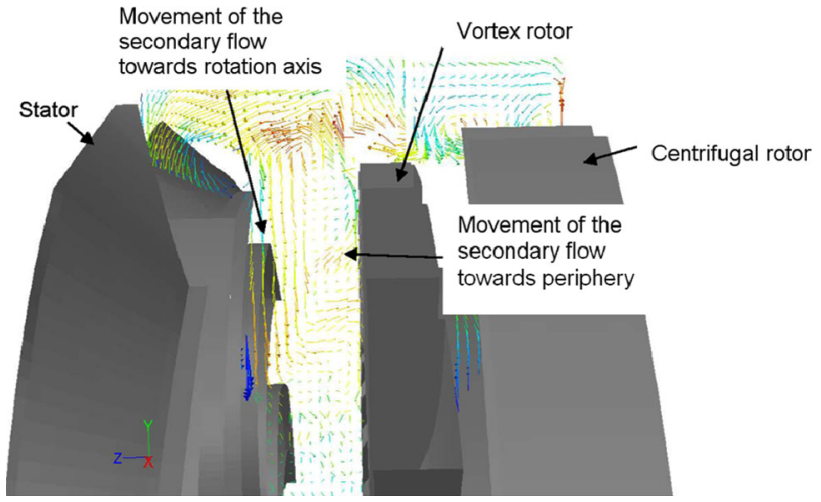

Fig. 16 Movement of the secondary fluid flow, $k_{L}=60$

comes from vortex rotor can be seen. Part of the main flow is taken by the vortex rotor, which then creates a secondary flow.

Looking at Fig. 16, it can be concluded that part of the main flow that comes out from the centrifugal rotor blades approaching the stator loses its kinetic energy, and fails to enter into the stator; instead, it gets pushed down (towards lower pressure) from the inlet in the stator to the axis of rotation (back flow). At that time, the secondary flow is created from this part of main fluid flow. When this secondary fluid flow arrives to the root of the vortex rotor blades, they take it and push it to the periphery (towards larger radius) by centrifugal force (Fig. 16). Given that the movement toward larger radius increases the angular velocity of the secondary flow, its kinetic energy increases. With the increased kinetic energy, the secondary flow collides with the main flow, transferring additional kinetic energy to it. The main flow that increased energy enters into the stator, ultimately generating an increased head of centrifugal vortex pump relative to the centrifugal pump.

5.7 Analysis of the Coherent Structures on the Vortex Rim. While summing up all the energy generation and losses, during this research, it was concluded that vortex rim transfers a larger quantity of energy to the main fluid flow coming from the centrifugal rotor then it can do only by giving additional kinetic energy to the secondary fluid flow (Sec. 5.6). From this research, it can be seen that there is another energy transfer mechanism at work. Figure 17 depicts the large scale structures found in the boundary layer at the vortex rim that are thought to play a significant role in the production and transport of turbulent energy and

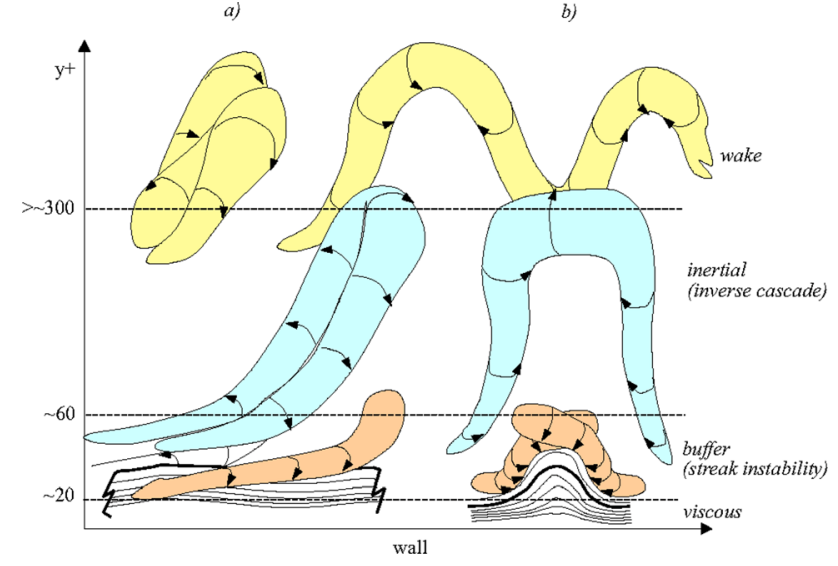

Fig. 17 Scheme of the coherent structures in a boundary layer: (a) side view and (b) front view with respect to flow direction

momentum to the main fluid flow, as experimented by Perry and Marusic [19].

Since most of the turbulent energy is contained in the largest scale eddies and the eddy size is limited by the distance from the wall, the near-wall energy containing eddies will be small while those centered in the core will be large. The transfer of energy from the small to large scales is an example of an inverse energy cascade as opposed to the classical energy cascade [20]. Since the constant shear stress in the logarithmic layer implies a constant momentum flux, the transfer is as much of momentum as of energy.

Figure 18 shows the streamlines over vortex rim with the $\mathbf{Q}$ criterion levels superimposed. The $\mathbf{Q}$ criterion represents the definition of "low-pressure tubular formations." Since the pressure difference is highest in the vortex core, these "low-pressure tubular formations" actually show core coherent structure. Q criterion is defined as I Eq. (3),

$$
\mathbf{Q}=\frac{1}{2}\left(\|\mathbf{W}\|^{2}-\|\mathbf{S}\|^{2}\right)
$$

where $\mathbf{Q}$ is the second tensor invariant, and $\mathbf{W}$ is the antisymmetric part of velocity gradient tensor, as shown by Jeong and Hussain [21].

Figure 18 shows the vortex rim being a generator of a large number of the coherent structures, in comparison to the centrifugal rotor back side. In addition, at the vortex rim, there are lots of streamlines forming a closed curve or a spiral pattern, as talked about by Villiers [22]. Streamlines at the outer diameter of the
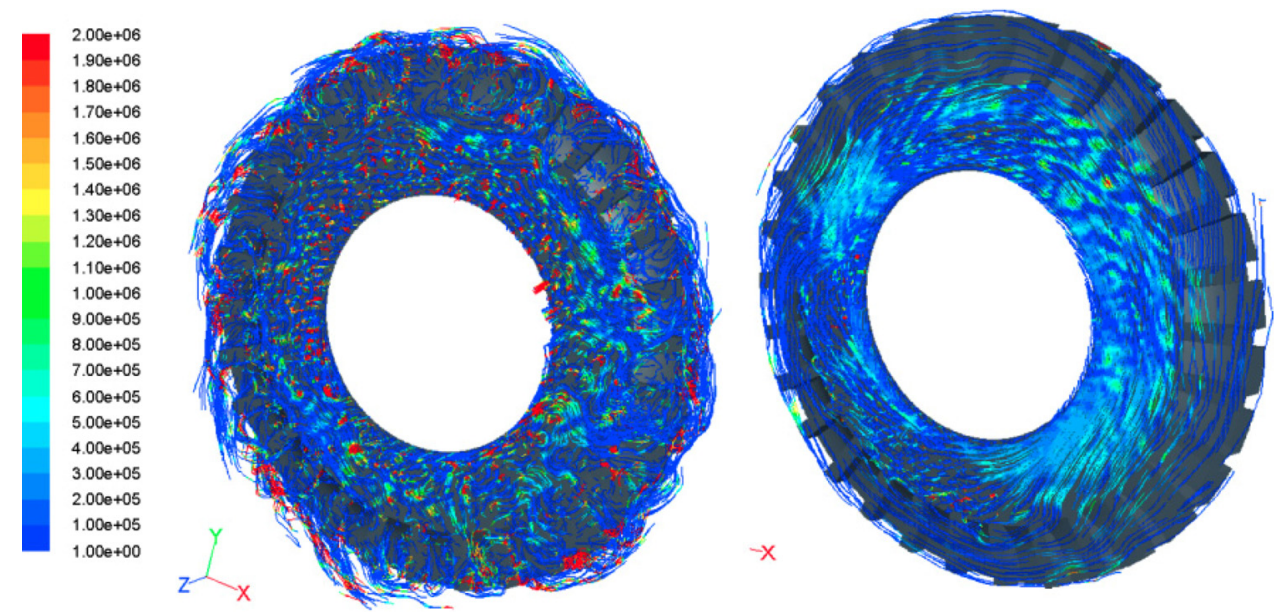

Fig. 18 Streamlines over back side of the centrifugal vortex rotor (left) and centrifugal rotor (right) colored by the $Q$ criterion

011002-6 / Vol. 135, JANUARY 2013

Transactions of the ASME 
centrifugal rotor are nearly concentric circles, with very low values of the $\mathbf{Q}$ criterion. Approaching to the axis of rotation of the centrifugal pump, the value of the $\mathbf{Q}$ criterion increases and the streamline loses its concentricity and some vortices are created. Approaching the rotational axis, the gap between the stationary stator wall, Fig. 1, and moving rotor wall, reduces; this represents a flow between two disks. Such a flow describes the Tesla pump / turbine, as described by Rice [23]. These vortices occur alternately. This periodicity is a property of Taylor vortices, as described by Taylor [24]. This represents the major difference between the left and right part of Fig. 18. It can be concluded that the vortex rim of the centrifugal vortex pump generates coherent structures, while at the back side of the centrifugal rotor of the centrifugal pump, Taylor vortices are generated.

Part of the coherent structure's energy can be transferred to the fluid flow and used for generating higher head, as opposed to the centrifugal pump where Taylor vortices are created. Those Taylor vortices cannot transfer their energy to fluid flow.

\section{Conclusion}

This work has proven and quantified the benefits of the centrifugal vortex pump stage in relation to the centrifugal one. The method used was based on the unsteady CFD experiments and experiments on physical models. Detailed measurements on the physical models of the power consumed to drive the centrifugal and the centrifugal vortex pump stage resulted in a more accurate quantification of energy balance.

It was proven that the kinetic energy of coherent vortex structures created by the vortex rim is added to the fluid flowing from the passages of the centrifugal rotor and thus increasing the total energy of the fluid before entering the stator. Furthermore, this extra energy is added to the main fluid stream by the longitudinal vortices generated by the edges of the vortex rim due to a change of kinetic energy of eddies and by the radial vortices detached from vortex rim vanes.

Centrifugal vortex pumps generate an increased head for a maximum of $23.13 \%$ (average increase of $11.64 \%$ ) compared to the centrifugal pumps with the same geometry at the same angular velocity. Furthermore, the vortex rim of the centrifugal vortex pump increases the amount of the head for working range from 0 to 0.57 $Q_{\max }$. At higher flow rates, it does not affect the level of head. Also this research has shown that adding vortex rotor to the centrifugal one improves the character of $Q-H$ characteristics which always has a positive slope as opposed to the characteristics of centrifugal pump, increasing the stability of the pump.

It has been found that vortex rotor head is $210 \%$ higher when operating in synergy with centrifugal impeller than when acting alone.

The efficiency of the centrifugal vortex is increased for the working regime 0 to $0.57 Q_{\max }$, while at the higher flow rates, efficiency is actually lower than that of the centrifugal pump. It can be concluded that at higher flow rates, the centrifugal vortex pump wastes driving energy in the spinning vortex rim. At lower flow rates, the contribution of the vortex rotor to the head is significant while it does not consume additional driving energy. This suggests that there must be some mechanism that recovers a part of the energy that otherwise is lost in centrifugal pumps. This mechanism has not yet been fully determined and represents energy transformation from small scales eddies to larger ones. These attached vortices are the chief turbulent energy and momentum transfer mechanism in the inertial layer (if the Re number is high enough for one to exist). Although it has not been confirmed, it is likely that the undulating spanwise vortices found in the wake region are the product of the largest hairpin eddies that eventually detach from the wall and merge with each other.

In further research, the effect of adding the vortex rim to the centrifugal pump stage to the cavitation should be investigated. It is necessary to carry out a detailed study of the impact of the shape of the vortex rim vanes and of the dimensions of the vortex chamber on the characteristics of the centrifugal vortex pump.

\section{References}

[1] Mihajlovič, P. O., Jurevič, M. I., Borisovič, K. P., Isaakovič, R. A., and Pavlovič, T. I., 2001, "New Rotary-Vortex Pumps for Crude Oil Production in the Complicated Conditions," Proceedings of the 1st International Conference of Technical Sciences, Voronež, Russia, pp. 128-143.

[2] Isaakovič, R. A., and Vasiljevič, G. N., 2003, "High-Head, Economical Modification of Multistage Centrifugal Pump for Oil Production," Proceedings of the 2nd International Conference of Technical Sciences, Voronež, Ruissa, pp. 221-230.

[3] Karakulov, S. T., Meljnikov, D. J., Pereljmman, M. O., and Dengaev, A.V., 2005, "Ways of Increasing Efficiency of Oil Exploitation From Oil Wells", Proceedings of the International Conference of Technical Sciences, Voronež, Russia, pp. 268-279.

[4] Melzi, E., 2008, "Vortex Impeller for Centrifugal Fluid-Dynamic Pumps," European Patent Application No. EP1 961965 A2.

[5] Franjić, K., 1996, Measurements in Mechanics of Fluid, Engineering Handbook IP1, University Press, Zagreb, Croatia, pp. 1015-1034.

[6] Goldstein, R. J., 1996, Fluid Mechanics Measurements, 2nd ed., Taylor \& Francis, New York.

[7] Upp, E. L., and LaNasa, P. J., 2002, Fluid Flow Measurement a Practical Guide to Accurate Flow Measurement, 2nd ed., Gulf Professional Publishing, Woburn, MA.

[8] ISO 5167-1:2003, "Measurement of Fluid Flow by Means of Pressure Differential Devices, Part 1: Orifice Plates, Nozzles, and Venturi Tubes Inserted in Circular Cross-Section Conduits Running Full."

[9] Bean, H. S., ed., 1971, Fluid Meters Their Theory and Application, 6th ed., ASME, New York.

[10] EN 837-2:1998, "Pressure Gauges, Part 2: Selection and Installation Recommendations for Pressure Gauges."

[11] Spalart, P. R., 2009, "Detached-Eddy Simulation," Ann. Rev. Fluid Mech., 41(1), pp. 181-202.

[12] Travin, A., Shur, M., Strelets, M., and Spalart, P. R., 2002, "Physical and Numerical Upgrades in the Detached-Eddy Simulation of Complex Turbulent Flows," Advances in LES of Complex Flows, Kluwer Academic Publishers, Dordrecht, The Netherlands, pp. 239-254.

[13] ANSYS Inc., 2009, "ANSYS FLUENT 12.0 Theory Guide," Ansys, Ann Arbor, MI.

[14] Dochterman, R. W., 1976, "Centrifugal-Vortex Pump," General Electric Company, U.S. Patent No. 3,936,240.

[15] Lobanoff, V. S., and Ross, R. R., 1992, Centrifugal Pumps-Design \& Application, 2nd ed., Butterworth-Heinemann, Houston, TX.

[16] Mihalić, T., Guzović, Z., and Sviderek, S., 2011, "Improving Centrifugal Pump by Adding Vortex Rotor," J. Energy Technol., 4(2), pp. 11-20.

[17] Gülich, J. F., 2008, Centrifugal Pumps, 2nd ed., Springer-Verlag, New York.

[18] Matijašević, B., Sviderek, S., and Mihalić, T., 2006, "Numerical Investigation of the Flow Instabilities in Centrifugal Fan," Proceedings of the WSEAS Conference on Fluid Mechanics and Aerodynamics, Agios Nicolaos, Greece, p. 5.

[19] Perry, A. E., and Marusic, I., 1995, "A Wall-Wake Model for the Turbulent Structure of Boundary Layers-Part 1: Extension of the Attached Eddy Hypothesis," J. Fluid Mech., 298, pp. 361-388.

[20] Kolmogorov, A. N., 1991, "The Local Structure of Turbulence in Incompressible Viscous Fluid for Very Large Reynolds Numbers," Dokl. Akad. Nauk., 30, pp. 301-305.

[21] Jeong, J., and Hussain, F., 1995, "On the Identification of a Vortex," J. Fluid Mech., 285, pp. 69-94

[22] Villiers, E., 2006, "The Potential of Large Eddy Simulation for the Modeling of Wall Bounded Flows," Ph.D. thesis, Imperial College of Science, Technology and Medicine, London, UK.

[23] Rice, W., 1991, "Tesla Turbomachinery," Proceedings of the 4th International Nikola Tesla Symposium, Belgrade, Serbia, pp. 117-125.

[24] Taylor, G. I., 1923, "Stability of a Viscous Liquid Contained Between Two Rotating Cylinders," Philos. Trans. R. Soc., 223, pp. 289-343. 